Quim. Nova, Vol. 33, No. 4, 994-999, 2010

\title{
EL APRENDIZAJE BASADO EN PROBLEMAS COMO ESTRATEGIA PARA EL CAMBIO METODOLÓGICO EN LOS TRABAJOS DE LABORATORIO
}

\author{
Juan-Antonio Llorens-Molina \\ Departamento de Química, Escuela Técnica Superior del Medio Rural y Enología, Universidad Politécnica de Valencia, Camino de \\ Vera s/n, 46021 Valencia, España
}

Recebido em 5/5/09; aceito em 30/11/09; publicado na web em 26/3/10

\begin{abstract}
PROBLEM BASED LEARNING AS ESTRATEGY FOR METHODOLOGICAL CHANGE IN LABORATORY WORK. The Problem Based Learning (PBL) can be used as a strategy for methodological change in conventional learning environments. In this paper, the integration of laboratory work in PBL grounded activities during an introductory organic chemistry course is described. The most decisive issues of their implementation are discussed. The results show how this methodology favours the laboratory work contextualization in subject-matter and promotes the Science-Technology-Society-Environment relationships. Besides, it contributes to competence development like planning and organization skills, information search and selection, cooperative work, etc., the same way as the tutorial action improvement.
\end{abstract}

Keywords: problem based learning; laboratory work; competences.

\section{INTRODUCCIÓN Y OBJETIVOS}

\section{El aprendizaje basado en problemas}

El aprendizaje basado en problemas (ABP) es una metodología educativa ampliamente extendida actualmente a numerosos campos de conocimiento. ${ }^{1}$ Como estrategia curricular institucional puede ser concebido como eje vertebrador de todo el proceso de aprendizaje, ${ }^{2}$ pero también puede ser útil, desde la innovación docente, para la transformación de actividades convencionales como los clásicos ejercicios, problemas o trabajos de laboratorio en tareas que favorezcan la adquisición de competencias de carácter metodológico y comunicativo, ${ }^{3}$ así como la incorporación de las tecnologías de la información y la comunicación. ${ }^{4,5}$

Esencialmente, el ABP trata de introducir al alumnado en el contexto de un problema vinculado a la realidad social y profesional de modo que deba planificar, mediante un trabajo cooperativo y bajo la supervisión del profesorado, un proceso de resolución que implica las siguientes etapas: la exploración del problema y la generación de hipótesis; la identificación de los conocimientos necesarios para abordarlos; la búsqueda de información y estudio independiente del tema; el análisis crítico y la discusión sobre los conocimientos adquiridos; la aplicación de éstos a la resolución del problema y la reflexión sobre el proceso, llevando a cabo el feed-back necesario.

Desde un punto de vista teórico, el ABP se fundamenta en las tendencias educativas más relevantes de las últimas décadas, tales como las diferentes aportaciones de la psicología cognitiva. Así, se considera esencial el papel de los conocimientos previos ya que éstos determinan el volumen y la naturaleza de los que el alumno va integrando posteriormente en su proceso de aprendizaje, siendo continuamente activados y reestructurados a medida que se avanza en el análisis y resolución del problema. De este modo, el aprendizaje puede contemplarse como la construcción de un entramado conceptual progresivamente más rico y complejo que exige la incorporación de nuevas herramientas metodológicas como las características de la actividad científica: formulación de hipótesis, elaboración de diseños experimentales, etc. Consecuentemente, el ABP puede constituir un marco privilegiado para desarrollar un aprendizaje concebido como investigación dirigida. ${ }^{6}$

\footnotetext{
*e-mail: juallom2@qim.upv.es
}

Asimismo, el ABP puede considerarse una concreción de la orientación constructivista del aprendizaje. ${ }^{7}$ En efecto, el alumnado asume responsabilidades en la identificación de aquello que necesita aprender y en la búsqueda de estrategias para obtener y aplicar los conocimientos. Además, este proceso tiene lugar en el marco de la interacción social con sus iguales y con el profesorado, cuyo papel es el de facilitador y creador del contexto adecuado para el aprendizaje autónomo de sus alumnos. Desde estos fundamentos teóricos, puede entenderse la importante contribución que el ABP puede aportar al desarrollo de las competencias genéricas, aspecto esencial en la construcción del Espacio Europeo de Educación Superior, principal reto en nuestro actual contexto educativo. De acuerdo con las orientaciones del documento: Tuning Educational Structures in Europe, ${ }^{8}$ algunas de dichas competencias son: el desarrollo de la autonomía y de la capacidad de autorregulación; la adquisición de habilidades comunicativas y de trabajo cooperativo; las destrezas relacionadas con la búsqueda, procesado y selección de la información y la capacidad para contextualizar el contenido de las asignaturas desde una perspectiva ciencia-tecnología-sociedad-medio ambiente. Por otra parte, los resultados de diferentes investigaciones muestran también que el ABP no solamente contribuye al desarrollo de competencias genéricas, sino que mejora la persistencia y solidez del aprendizaje de conceptos. ${ }^{9}$

En cuanto al aprendizaje de la química, existen numerosas y variadas propuestas fundamentadas o cercanas al ABP, generalmente con un enfoque experimental. ${ }^{10}$ En ellas, esta metodología se propone como una estrategia de cambio metodológico y de integración del trabajo de laboratorio en el currículo, ${ }^{11}$ y al mismo tiempo se justifica su contribución al desarrollo de habilidades cognitivas de alto nivel. ${ }^{12}$

En lo concerniente a su puesta en práctica, Duch describe detalladamente un modelo de aplicación del ABP en la enseñanza universitaria de la química incluyendo el modelo de curso, el ciclo de aprendizaje, la gestión del trabajo cooperativo y la evaluación. ${ }^{13}$ Diferentes aportaciones se refieren a la propuesta de actividades concretas, ${ }^{14}$ más próximas a los estudios de caso, ${ }^{15} \mathrm{y}$ centradas algunas en aspectos socioeconómicos y profesionales. ${ }^{16}$ Otras contribuciones se refieren a campos específicos de la química; por ejemplo, en química orgánica cabe citar el manual de actividades experimentales de Lehman, ${ }^{17}$ así como la introducción en esta rama de la química de metodologías activas mediante actividades fundamentadas en el ABP. ${ }^{18}$ Asimismo, también han sido descritas aplicaciones específicas en la química analítica y medioambiental. ${ }^{19}$ Por 
último, otro conjunto de investigaciones se centran en la incorporación de las nuevas tecnologías al ABP.,20

\section{Objetivos}

El objetivo de esta investigación ha sido el diseño, experimentación y evaluación de actividades basadas en el ABP como estrategia para favorecer la adquisición de competencias genéricas en un curso introductorio de química orgánica. Partiendo de las competencias ya citadas anteriormente, ${ }^{8}$ la finalidad de estas actividades es: contribuir a una mejor planificación y organización de las tareas de los estudiantes en un contexto de trabajo cooperativo; promover en ellos conductas autónomas, tanto referidas al desempeño de las tareas de laboratorio, como a la potenciación de su actividad metacognitiva; ${ }^{21}$ y contextualizar los contenidos teóricos y experimentales del curso estableciendo relaciones con problemas relevantes en el ámbito agroalimentario y de la ingeniería rural. ${ }^{22}$ En este sentido, debe tenerse en cuenta que el ABP, al desarrollarse en escenarios inspirados en la vida real, es esencialmente multidisciplinar y ello exige un esfuerzo de integración y coordinación con el resto de materias del curso y de la titulación.

El proceso de experimentación ha tenido lugar durante tres cursos consecutivos, con un número total de 18 problemas (enumerados en el material suplementario, $1 \mathrm{~S}$ ) e incorporando progresivas innovaciones como consecuencia del feed-back obtenido. Las actividades basadas en dichos problemas han sido desarrolladas voluntariamente en grupos de trabajo cooperativo. Cuando éstos estaban formados total o mayoritariamente por alumnos que cursaban la asignatura por primera vez, los problemas asignados fueron seleccionados de modo que las prácticas de laboratorio ordinarias (obligatorias) respondieran a las necesidades formativas identificadas en el planteamiento del problema; en el caso de los grupos formados por repetidores (exentos de realizar las prácticas), los problemas han estado más vinculados a los contenidos teóricos, pudiendo incluir también actividades experimentales.

La evaluación de las actividades propuestas se ha centrado en el análisis evolutivo de algunos aspectos especialmente relevantes como: su iniciación; el modo en que el alumnado se incorpora y participa en ella; la evaluación de las propias actividades en cuanto a su capacidad para implicar a los estudiantes; la conciliación entre el desarrollo de la autonomía en el alumnado y el papel facilitador y orientador del profesorado; la manera de proporcionar feed-back a lo largo del proceso; la selección de los métodos de evaluación más adecuados; la gestión del trabajo cooperativo y la percepción de los estudiantes acerca de los aspectos anteriormente citados.

\section{DESCRIPCIÓN DE LA PROPUESTA DIDÁCTICA}

\section{Contexto de la experimentación}

Nuestra propuesta didáctica ha estado enmarcada en la asignatura Fundamentos Químicos de la Ingeniería del primer curso de la Escuela Técnica Superior del Medio Rural y Enología, en la Universidad Politécnica de Valencia (España). Ésta se ha aplicado a lo largo de un cuatrimestre ( $60 \mathrm{~h}$ lectivas entre clases teóricas y de laboratorio) dedicado a la introducción de la química orgánica, tras otro similar, dedicado a la química general. Desde el punto de vista metodológico, la asignatura se imparte mediante 2 sesiones semanales de 90 min que integran periodos de exposición y discusión relativamente breves, con ejercicios de aplicación individuales y colaborativos. Por otra parte, y ello es importante a la hora de considerar la viabilidad de nuestra propuesta, de las $6 \mathrm{~h}$ semanales dedicadas a la acción tutorial, unas 4 se han dedicado al seguimiento de los grupos del trabajo y a la planificación y ejecución de los experimentos de laboratorio que, al margen del programa ordinario de prácticas de laboratorio, vienen exigidos por el desarrollo de los problemas. En cuanto a la evaluación sumativa, los contenidos teóricos son valorados en un $70 \%$ y las prácticas de laboratorio en un $30 \%$. Una vez obtenida la calificación global del curso, si ésta es superior a 4, la calificación de la actividad basada en el ABP puede aumentar hasta en un 30\% dicha calificación global.

Desde el punto de vista contextual también conviene hacer referencia al hecho de que nuestra universidad se halla en pleno proceso de incorporación al Espacio Europeo de Educación Superior, lo que implica la puesta en práctica de innovaciones que afectan sensiblemente a la metodología y práctica de la evaluación. Simultáneamente, coexisten normas académicas tales como el derecho a un examen global de la asignatura sin otro requisito que hallarse matriculado. Ello da lugar a dos colectivos de estudiantes bien diferenciados: los que asisten habitualmente a clase, que de manera generalizada han participado en la experiencia y los alumnos repetidores o con actividad laboral permanente, que hacen uso del derecho a dicho examen y se han implicado en mucha menor medida.

\section{Marco curricular}

El programa de la asignatura responde a los contenidos habituales de cualquier curso introductorio a la química orgánica, desde la perspectiva de una ingeniería relacionada con la agricultura y el medio rural. Tanto el programa teórico como las prácticas de laboratorio son detallados en el material suplementario.

En su desarrollo podemos distinguir algunos objetivos básicos, de índole conceptual, como elementos estructurantes que le aportan unidad y coherencia: la comprensión de los aspectos generales de la reactividad de las diferentes funciones orgánicas a partir de la naturaleza de los enlaces químicos presentes, justificando así cómo determinadas estructuras dan lugar a reacciones específicas que permiten su identificación; la capacidad para explicar las propiedades físicas a partir de las fuerzas intermoleculares y la naturaleza de éstas, a su vez, como consecuencia de la estructura molecular; comprender cómo estas propiedades son el fundamento de los métodos de separación (destilación, extracción, cromatografía, etc.) y, por último, valorar el papel de la composición química y reactividad de las sustancias como elementos decisivos en el análisis económico y medioambiental de numerosos procesos tecnológicos.

Por lo que respecta al programa ordinario de prácticas, éstas tienen como finalidad prioritaria proporcionar al alumnado, con escasa o nula formación experimental previa, un conjunto de técnicas básicas de laboratorio centradas principalmente en los métodos de separación ya citados, así como apoyar la adquisición de ciertos contenidos teóricos. El trabajo en el laboratorio se desarrolla en parejas con una periodicidad semanal en sesiones de dos horas, según un calendario preestablecido. Desde los objetivos de la investigación, no se consideró conveniente modificar esta estructura, sino adaptarse a ella, tanto por razones organizativas como por el hecho de tratarse de prácticas de carácter muy básico y general.

Inmediatamente antes de cada sesión de laboratorio los estudiantes deben realizar un test online que se apoya en una presentación audiovisual a la que se accede dentro de la plataforma de docencia en red PoliformaT (proyecto Sakai) ${ }^{23} \mathrm{El}$ material audiovisual correspondiente es de disposición pública en http://dspace.upv.es/manakin/ y puede accederse al mismo a través del nombre del autor.

\section{Principios generales del diseño y aplicación de las actividades experimentadas}

En la aplicación del ABP en contextos de aprendizaje convencionales, podemos distinguir algunos aspectos clave relativos al diseño y ejecución de las actividades: su puesta en marcha; su gestión, por 
lo que respecta a la necesidad de regular el feed-back proporcionado y la evaluación de la actividad y su contribución a la calificación de la asignatura.

\section{El inicio de la actividad}

La selección de un escenario adecuado y su concreción en una actividad inicial o primera formulación del problema exige analizar cuidadosamente las relaciones entre los contextos social y profesional, por una parte, y académico por otra. Consecuentemente, se ha procurado partir de un análisis de la realidad más próxima relacionada con el sector agroalimentario y la ingeniería rural así como de los problemas medioambientales implicados. Desde el punto de vista académico se han considerado varios factores: los conocimientos y habilidades previos de todo tipo; las competencias genéricas y específicas propias de la titulación y la carga de trabajo que supone la actividad, que debe estar adecuadamente ponderada en el conjunto de la asignatura.

Esta actividad inicial ha sido uno de los aspectos más controvertidos y sometidos a revisión a lo largo de la experimentación. Aunque en la literatura se plantean estrategias más abiertas, tipo brainstorming, ${ }^{4} \mathrm{se}$ ha optado por una orientación inicial más directiva por dos razones: en primer lugar, por el bajo nivel general de conocimientos previos; en segundo, porque se trata de actividades que en su mayor parte requieren trabajos de laboratorio, sometidos a restricciones derivadas de la disponibilidad de horario, material y equipos, condiciones de seguridad, etc.

En el diseño de esta actividad inicial pueden distinguirse dos aspectos: el explícito, que constituirá el enunciado proporcionado al estudiante como punto de partida para su trabajo, y el implícito, relacionado con todas aquellas consideraciones que el profesorado debe tener en cuenta y que se refieren, principalmente, a los puntos de anclaje en la experiencia previa del alumno, cotidiana o académica. Estas consideraciones deben reflejarse en una hipótesis inicial acerca de las dificultades con que previsiblemente los estudiantes han de enfrentarse en los primeros pasos de la resolución del problema. Esta hipótesis inicial ha de ser posteriormente extendida a todo el proceso, tal como se describe en el ejemplo expuesto en el material suplementario, sobre una actividad relacionada con la fabricación del biodiesel (Figura 1S, material suplementario).

Podemos formular además dos importantes criterios de carácter práctico relativos a la puesta en marcha de cada actividad: la necesidad de orientar la entrevista inicial para proporcionar a los miembros del grupo la oportunidad de realizar alguna aportación positiva por modesta que sea, y que dicha entrevista dé lugar al compromiso con un plan de trabajo distribuido en tareas concretas.

El diseño de esta fase fue modificado en el segundo y tercer curso de experimentación incluyendo una primera tarea no presencial según un modelo próximo a las webquest, como punto de partida para la primera entrevista. ${ }^{24}$ La evolución de estas actividades iniciales puede observarse a través de algunos ejemplos en: https://poliformat.upv.es/ access/content/user/19877419/Actividades iniciales en ABP_Explotaciones Agropecuarias_.doc

\section{El desarrollo de la actividad}

El proceso global de la actividad y su integración en el contexto del curso viene expuesto en la Figura 1. Cabe indicar que dicho proceso fue objeto de importantes modificaciones a lo largo de la experimentación. Éstas afectaron principalmente a: la necesidad de proporcionar un mayor feed-back a los estudiantes durante el proceso, introduciendo una evaluación previa de la propuesta final, la gestión del trabajo cooperativo, aumentando el número de entrevistas con el grupo y llevando a cabo un mayor control del trabajo individual y al modo de exposición y evaluación final.

En el diagrama de la Figura 1 se muestra cómo la actividad inicial debe dar lugar a la identificación de aquellos conocimientos necesarios

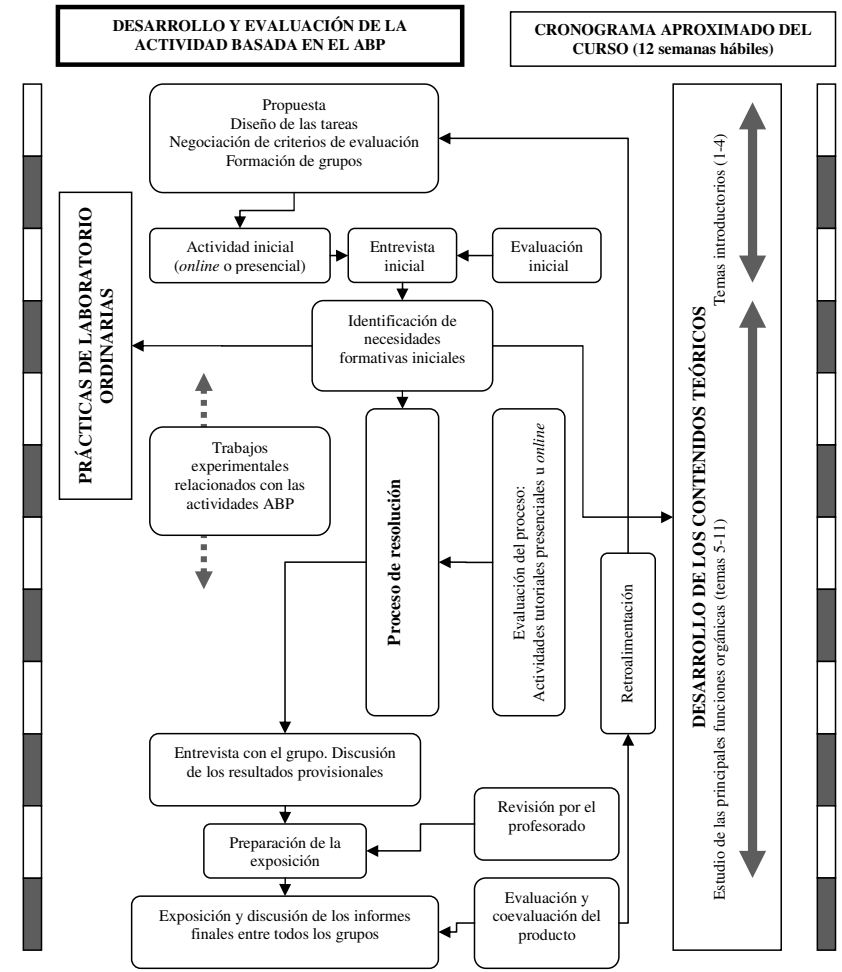

Figura 1. Desarrollo de las actividades fundamentadas en el ABP, dentro del contexto del curso

para abordar el problema y de las fuentes de información requeridas. El proceso de resolución implica también, obviamente, el trabajo personal de los estudiantes, integrando las tareas relacionadas con el desarrollo de las diferentes unidades didácticas y con el cumplimiento del programa de prácticas de laboratorio. Durante el desarrollo de cada actividad fue decisiva la atención a los grupos mediante la tutoría. Ésta incluyó dos entrevistas preceptivas: la inicial, a la que ya nos hemos referido y otra, previa a la elaboración del producto final. También se llevó a cabo un número variable de entrevistas para el seguimiento del trabajo, aclarar dudas, plantear posibilidades, etc., teniendo como objetivo prioritario estimular el análisis crítico y la discusión en grupo sobre los conocimientos adquiridos. En esta interacción profesorado-grupos, la plataforma poliformaT, a la que ya hemos hecho referencia, fue adquiriendo un importancia creciente durante el proceso de experimentación.

Por último, en cuanto a la fase final de la resolución, conviene precisar dos objetivos importantes: los estudiantes deben valorar críticamente en qué medida los resultados obtenidos dan respuesta al problema inicialmente planteado y alcanzar una síntesis integradora de los conocimientos adquiridos con los del resto de la asignatura.

Un elemento clave para la gestión de la actividad: la elaboración de una hipótesis de trabajo sobre el proceso de resolución del problema

La gestión de una actividad basada en el ABP exige conciliar la autonomía del estudiante y el papel directivo del profesor. En otras palabras, el papel de éste como facilitador del proceso de aprendizaje exige, por una parte, plantear dificultades y contradicciones, analizar críticamente las propuestas de los estudiantes, considerar qué información debe o no debe proporcionar en cada momento, etc., para estimular los procesos de autorregulación en el alumno, generando ideas y propuestas alternativas. Por otra parte, es evidente que el proceso no puede interrumpirse, creando una situación de desorientación y desmotivación que los estudiantes no puedan asumir, por lo que es necesario proporcionar en cada momento el feed-back adecuado. Al mismo tiempo, hemos de tener en cuenta que este tipo de actividades 
deben ser muy abiertas desde la perspectiva del alumno, pero quizás no tanto desde la perspectiva del profesor. Éste, en buena medida, debe prever el desarrollo de la actividad, disponiendo de criterios y recursos para reorientarla. Éstos pueden concretarse en un documento que, a modo de hipótesis de trabajo, sirva de guía orientadora del proceso de resolución de cada problema, tal como el mostrado en la Figura 1S (material suplementario). Esta planificación debe apoyarse, por una parte, en la investigación didáctica, que puede ayudarnos a prever las principales dificultades conceptuales y metodológicas de los estudiantes y, por otra, no menos importante, en la propia experiencia docente.

\section{La evaluación}

La coherencia entre los modelos de evaluación y de aprendizaje es una exigencia ineludible en cualquier proceso de innovación metodológica y, particularmente, en el aprendizaje basado en problemas. ${ }^{25}$ En contextos de aprendizaje convencionales, la evaluación ha estado centrada habitualmente en la medición del rendimiento académico del estudiante. Por el contrario, el ABP exige evaluar el proceso de aprendizaje en sí mismo, con la multiplicidad de factores que lo afectan: organizativos, relaciones con el diseño de los recursos didácticos, con el papel del profesorado, etc. La estrategia de evaluación desarrollada es descrita en el diagrama de la Figura 2, en el que se relacionan los diferentes aspectos de la evaluación y los instrumentos utilizados. En él pueden distinguirse diferentes dimensiones:

Dimensión institucional, referida a su contribución a la calificación de la asignatura, a sus implicaciones en la normativa general de evaluación aplicada y a todos aquellos aspectos que aportan información relevante desde el punto de vista de la organización docente (horario, instalaciones, personal de laboratorio, etc.).

Dimensión social, vinculada al desarrollo de actitudes y a la adquisición de competencias relacionadas con el aprendizaje cooperativo.

Dimensión académica, relacionada con la adquisición de conceptos, métodos, destrezas y habilidades relacionados con el currículo de la asignatura. En este sentido, es importante precisar que las presentaciones de los grupos están disponibles a través de PoliformaT y deben ser objeto de estudio por todos los alumnos, ya que su contenido puede ser considerado en el examen. De este modo, el trabajo desarrollado por los grupos es incorporado al contenido global del curso, sirviendo como extensión o profundización de las unidades didácticas. Por otra parte, en cuanto a la calificación final de la actividad, el modelo al que se ha llegado tras los tres cursos de experimentación queda reflejado en la tabla 1. En él cabe destacar la aplicación de la coevaluación.

\section{RESULTADOS DE LA EXPERIMENTACIÓN}

Los resultados de la experimentación constituyen el fundamento para la evaluación de la propuesta didáctica. En ella se han considerado los progresivos cambios habidos en el diseño como consecuencia del feed-back obtenido así como su percepción por el alumnado.

La Tabla 2 muestra los resultados del citado feed-back. En torno a los mismos cabe hacer los siguientes comentarios: en conjunto, se ha observado una implicación generalizada y permanente en los alumnos que asisten habitualmente a clase; más irregular ha sido la participación de algunos repetidores que inicialmente se incorporaron a los grupos de trabajo, siendo muy acusadas las diferencias en ritmo y estilo de trabajo entre ambos colectivos de estudiantes. Un modo de abordar este problema durante el tercer curso fue constituir grupos exclusivamente formados por repetidores y asignarles problemas no vinculados al programa de prácticas de laboratorio, ya que estos alumnos están exentos de ellas. Es significativo también el hecho de que en este tercer año la obligatoriedad de la participación como condición para optar a

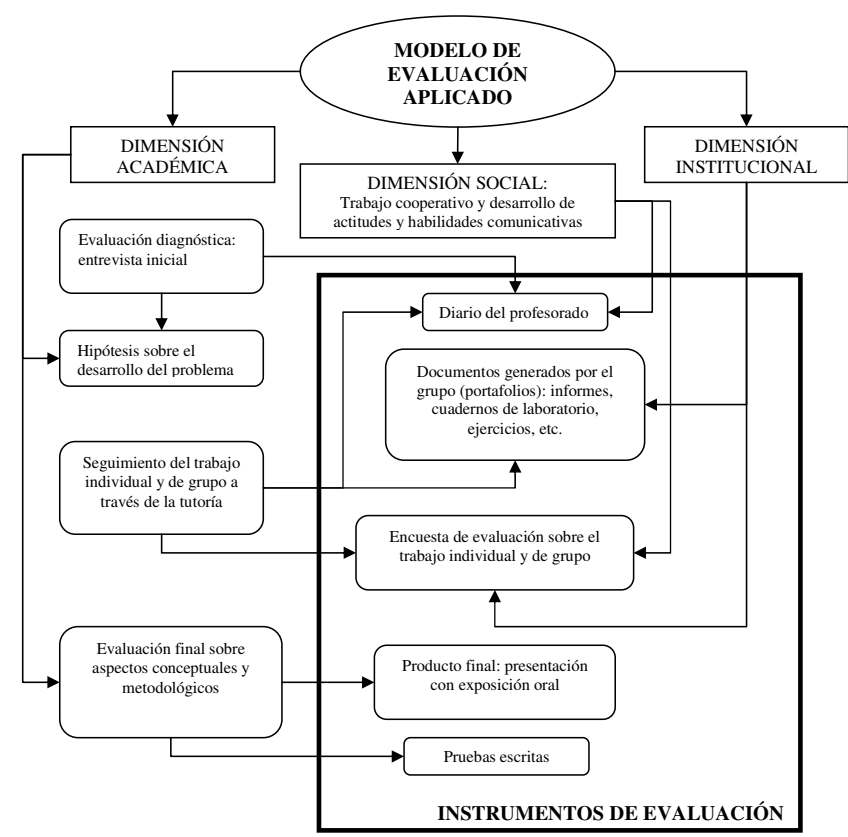

Figura 2. La evaluación de las actividades basadas en el ABP

Tabla 1. Criterios para la calificación del producto final de las actividades

Producto (presentación oral y debate)

\begin{tabular}{lll}
\hline Coevaluación & Valoración por el profesorado & Total \\
\hline
\end{tabular}

Interés Adecuación a Funda- Calidad Proceso:

Aspectos las normas y mentación de las Autono-

formales convenciones: teórica. fuentes de mía y

Claridad expresión de Corrección información creatividad

magnitudes y conceptual utilizadas (*)

unidades.

\begin{tabular}{lrrrrr}
\hline 10 & 3 & 4 & 3 & 10 & 30 \\
\hline$(*)$ Evaluable a partir del cuaderno del profesor & & &
\end{tabular}

un sistema de evaluación continua diera lugar a que todos los grupos finalizaran y presentaran su trabajo.

Otro aspecto importante del feed-back obtenido es la progresiva reducción del número de problemas puestos en juego. Si observamos la evolución mostrada en $1 \mathrm{~S}$ (material suplementario) podemos apreciar algunos aspectos de interés en cuanto a los motivos por los que se decidió descartar unos e incorporar otros: la escasa aportación del problema al contenido de la asignatura, bien por su excesiva complejidad o por la pobreza teórica de su desarrollo, al dar lugar a simples comprobaciones experimentales; la dificultad en el tratamiento experimental, ya que ciertas técnicas no admiten simplificaciones que sean compatibles con resultados claros que permitan abordar el problema con el rigor necesario; la propia formulación del problema, que da lugar a un planteamiento excesivamente puntual que no exige al grupo un desarrollo posterior de la suficiente entidad. Por otra parte, en algunas de las actividades el primer año el grado de conexión con la vida real y el carácter multidisciplinar no mostró ser lo suficientemente evidente y, de hecho, fueron eliminadas o reformuladas.

La percepción por el alumnado ha sido evaluada a través de una encuesta aplicada al final del primer y tercer curso. Dicha encuesta y sus resultados son mostrados en el material suplementario (Tabla 2S). Los ítems mejor valorados han sido los relacionados con el diseño, interés y adecuación de las actividades y la atención dedicada en la tutoría. En otros aspectos la valoración no ha sido tan claramente positiva, como sucede en relación a: la calidad del trabajo cooperativo, el grado de motivación - que no es claramente mayor que el del resto de actividades de la asignatura y la contribución de estas actividades al 
Tabla 2. Evolución del modelo de actividad a lo largo de los tres cursos de experimentación como consecuencia del feed-back obtenido

\begin{tabular}{|c|c|c|c|}
\hline & Curso 05/06 & Curso 06/07 & Curso 07/08 \\
\hline Participación & Voluntaria & Voluntaria & $\begin{array}{l}\text { Voluntaria pero vinculada a la posibilidad de } \\
\text { realizar exámenes parciales }\end{array}$ \\
\hline Duración total de la actividad & 6 semanas & 8 semanas & 10 semanas \\
\hline $\mathrm{N}^{\mathrm{o}}$ alumnos & 37 (14 grupos) & 13 (4 grupos) & 26 (11 grupos) \\
\hline
\end{tabular}

Formación de los grupos

Grupos espontáneos preferentemente de 3 miembros Grupos espontáneos prefe-
rentemente de 3 miembros

Elección/distribución de los temas

Elección entre una lista sugerida por el profesor por orden de formación del grupo. Posibilidad de aportar temas por iniciativa del grupo.

Evaluación del producto Valoración del cartel por el profesor en todos sus aspectos.

Elección entre una lista sugerida mucho más cerrada. Sin posibilidad de proponer nuevos problemas.

Presentación oral y debate. Coevaluación

$20 \%$ (mínimo de 4 en el resto de contenidos)

Aplicación en las convocatorias de junio y septiembre

Evaluación del proceso por los alumnos Encuesta

Grado de vinculación con las prácticas de laboratorio
$25 \%$ (mínimo de 4 en el resto de contenidos)

Aplicación en las convocatorias de junio y septiembre

Valoración en la sesión final de exposición de resultados

Total. Todos los problemas propuestos se hallan vinculados a prácticas.
Relativo. Un 50\% de los temas no está directamente relacionado con el programa de prácticas.
Grupos espontáneos de 3 miembros. Se distingue entre los grupos formados por alumnos que asisten a las prácticas y los formados por repetidores

Propuesta directa de dos temas a elegir. Se introducen algunos cambios: enfoques diferentes de un mismo problema para dos grupos.

Presentación oral y debate.

Coevaluación.

Establecimiento de una distribución fija de la puntuación en los diferentes aspectos

Aumento del $30 \%$ sobre la nota final (mínimo de 4 en el resto de contenidos)

Aplicación solamente a la convocatoria de junio.

Encuesta

Se vuelve a una distribución similar a la del primer año, pero separando claramente los grupos formados por asistentes a las prácticas, de los que no lo están. aprendizaje del resto de los contenidos, aunque en este caso se observó una clara mejoría el tercer curso probablemente por el establecimiento de relaciones más explícitas con los contenidos teóricos.

\section{CONCLUSIONES}

Como conclusión general, cabe considerar particularmente satisfactorio el impulso de la tutoría, efecto que se extendió progresivamente al resto de la asignatura. Las dificultades han surgido, principalmente, en el ámbito organizativo y de normativa académica por las causas ya citadas al describir el contexto de la experimentación. En este sentido, cabe esperar que el proceso de innovación asociado a la implantación de las nuevas titulaciones, dentro del Espacio Europeo de Educación Superior, traiga consigo una mayor coherencia entre normativa y principios metodológicos. Como conclusiones más específicas podemos distinguir algunas cuestiones que valoramos como particularmente críticas:

La propia naturaleza de los problemas - éste muestra ser, tal vez, el elemento más decisivo en el éxito de la actividad. En este sentido, es necesario un esfuerzo desde la investigación educativa para identificar situaciones problemáticas en contextos de interés que favorezcan el desarrollo de estrategias más investigativas, frente a la mera obtención, selección y organización de la información. Estos procesos requieren a su vez una atención específica, pero como parte de dichas estrategias, no como una finalidad en si misma. También es necesario que den lugar a tratamientos experimentales relativamente sencillos y previsibles, dadas las posibles dificultades a la hora de disponer del laboratorio y de su personal técnico al margen del horario habitual de prácticas. Una posible solución, en innovaciones como la expuesta en este trabajo, podría ser la reducción del número de problemas diferentes puestos en juego, introduciendo en cada uno diferentes versiones que garanticen la necesaria autonomía de cada grupo durante su desarrollo. Por ejemplo, el problema basado en el precio de los aceites esenciales (material suplementario) puede abordarse simultáneamente por varios grupos, seleccionando plantas aromáticas diferentes.
La coordinación entre el proceso de resolución del problema y el desarrollo de los contenidos teóricos y prácticos del curso - No puede olvidarse que uno de los rasgos específicos del ABP es precisamente el reconocimiento previo por el estudiante de qué necesita aprender para resolver el problema. Ello exige una cuidadosa planificación temporal del trabajo de cada grupo de modo que los conocimientos del curso respondan a las expectativas generadas y no constituyan una mera aplicación del aprendizaje adquirido. En este sentido, uno de los principales obstáculos con que puede enfrentarse una propuesta como la descrita en esta investigación es la posible falta de coordinación entre el calendario de prácticas y el desarrollo de los problemas, que en ocasiones puede llevar a una intervención excesivamente directiva en el trabajo de los grupos. Por otra parte, la necesidad de realizar actividades experimentales específicas en el desarrollo de cada problema supone un esfuerzo añadido en cuanto a la organización de recursos, aumentando todavía más, si cabe, la complejidad de la planificación de este tipo de actividades. No obstante, a juzgar por la valoración del alumnado, la coordinación entre el desarrollo del problema y el resto del curso ha sido razonablemente adecuada.

La regulación del ritmo de trabajo de los grupos - Las principales dificultades en este aspecto han sido: la falta de tiempo para organizar sesiones presenciales de trabajo cooperativo. La experimentación se ha apoyado excesivamente en el tiempo dedicado a la tutoría y ello ha dificultado el control de la dinámica interna de los grupos, en los que debe garantizarse una participación equilibrada. Por este motivo, en próximos cursos se asignará un tiempo, aunque sea mínimo, dentro de las clases ordinarias, para este tipo de actividades; el feed-back que se proporciona durante el proceso de resolución no llega a ejercer la suficiente presión para que el trabajo se realice siempre de un modo continuado. Particularmente, los grupos tienden a comenzar lo más tarde posible y, consecuentemente, a lo largo de los tres cursos, el inicio de la actividad fue adelantándose progresivamente, ampliando su duración. La causa puede radicar probablemente en el hecho de que la calificación de la actividad se lleva a cabo principalmente a partir de la exposición final, por lo que parece aconsejable que las evalua- 
ciones intermedias realizadas afecten sensiblemente a la calificación.

El planteamiento inicial de la actividad - También muestra ser un elemento decisivo, ya que determina considerablemente su desarrollo posterior. El lanzamiento de la actividad a través de una metodología próxima a la webquest ha mostrado ser extraordinariamente útil como elemento dinamizador y enriquecedor de la entrevista inicial.

El grado en que el alumnado es capaz de identificar de manera autónoma sus necesidades de aprendizaje frente a la resolución de un problema - Este objetivo se ha alcanzado en un grado bastante limitado. Ello puede ser debido a las siguientes causas: una, evidente en nuestro contexto educativo, es la escasa familiaridad de alumnos y profesores con este tipo de metodologías, otra, el hecho de que algunas de dichas necesidades formativas ya vienen muy definidas por la vinculación existente entre los problemas propuestos y el programa de prácticas de laboratorio, con todos los recursos educativos asociados al mismo: manual de prácticas, actividades pre-laboratorio, etc.

La naturaleza de las fuentes de información utilizadas - Buena parte de los estudiantes utilizan de manera casi exclusiva los buscadores generalistas de la web como fuente de información. De ahí se desprende la necesidad de incidir de manera específica en la capacidad de buscar y procesar la información, como competencia genérica. Concretamente, sería necesario: enseñar a usar de modo eficaz los recursos de la web; promover la diversificación en cuanto al tipo de fuentes de información y fomentar el análisis crítico de la misma.

Características del producto final solicitado a los estudiantes y su modo de evaluación - Este aspecto ha sido objeto de una profunda revisión en cuanto al tipo de producto solicitado a los grupos y modo de evaluarlo. En el diseño inicial se propuso la elaboración de un cartel, con un formato similar al habitualmente utilizado en jornadas y congresos, que era expuesto en el laboratorio. Este modo de proceder mostró varias insuficiencias importantes: la escasa participación e impacto en el resto del alumnado, dando lugar, en la práctica, a un modelo de evaluación excesivamente centrado en el profesor y la profusión de errores, en ciertos casos, de carácter conceptual. La sustitución del cartel por una presentación y discusión de cada trabajo en una sesión colectiva, a la que asisten obligatoriamente todos los participantes en la actividad, así como la introducción de la coevaluación, han permitido elevar el grado de participación del alumnado dando lugar también a un progresivo aumento de la calidad de sus aportaciones.

\section{MATERIAL SUPLEMENTARIO}

El material suplementario contiene los siguientes elementos: los programas de la asignatura (teoría y prácticas); la enumeración de los problemas experimentados a lo largo de los tres cursos (S1) con una breve justificación de las razones por las que algunos fueron excluidos; la descripción detallada de un ejemplo de actividad y una descripción más breve de algunos de los problemas cuyo desarrollo ha resultado de mayor interés; la encuesta aplicada a los estudiantes para la evaluación de la actividad, con sus resultados. Está disponible gratuitamente en http://quimicanova.sbq.org.br, en PDF.

\section{REFERENCIAS}

1. Ward, J. D.; Lee, C. L.; J. Fam. Consum. Sci. Educ. 2002, 20, 16.

2. http://www.face.aau.dk/face/pbl/aau_pbl.htm, accedida en Marzo 2009; http://www.udel.edu/pbl/, accedida en Marzo 2009.

3. Paulson, D. R.; J. Chem. Educ. 1999, 76, 1136; Northwood, M. D.; Northwood, D. O.; Northwood, M. G.; Global J. Eng. Educ. 2003, 7, 157; Mohd, K.; International Problem-Based Learning Symposium, Singapore, 2007; Kelly, O. C.; Finlayson, O. E.; Chem. Educ. Res. Pract. 2007, 8, 347; Gambhir, P. B.; International Problem-Based Learning
Symposium, Singapore, 2007, http://www.rp.sg/symposium/download/ International_PBL_Symposium2007.pdf, accedida en Marzo 2010.

4. Poikela, S.; Vuoskoski, P.; Kärnä, M.; International Problem-Based Learning Syposium, Singapore, 2007. (ver ref. 3).

5. Brodie, L.; Gibbings, P.; International Conference on Design Education, New South Wales: Sydney, 2007.

6. Gil, D.; Enseñanza de las Ciencias 1993, 11, 197; Folmer, V.; De Vargas Barbosa, N. B.; Soares, F. A.; Rocha, J. B. T.; Revista Electrónica de Enseñanza de las Ciencias 2009, 8, 232.

7. Hendry, G. D.; Frommer, M.; Walker, R. A.; Journal of Further and Higher Education 1999, 23, 359; http://crlt.indiana.edu/publications/ journals/TR16-01.pdf, accedida en Marzo 2009.

8. Mitchell, T. Y Whewell, R. En Tuning Educational Structures in Europe; Comisión Europea de Educación y Cultura, Ed. Bruselas, 2003, p. 111.

9. Anderson, W. L.; Mitchell, S. M.; Osgod, M. P.; Biochem. Mol.Biol. Educ. 2006, 33, 387; Tarhan, L.; Acar, B.; Res. Sci. Technol. Educ. 2007, 25 , 351; Gurses, A.; Acikyildiz, M.; Dogar, C.; Sozbilir, M.; Res. Sci. Technol. Educ. 2007, 25, 99; Tarhan, L.; Ayar-Kayali, H.; Urek, R. O.; Acat, B. Res. Sci. Educ. 2007, 38, 285; Downing, K. J.; Shin, K.; Kwong, T.; International Problem-Based Learning Symposium, Singapore, 2007; Dochy, F.; Segers, M.; Van den Bossche, P.; Gijbels, D.; Learn. Instr. 2003, 13, 533.

10. Mcdonnell, C.; O'Connor, C.; Seery, M. K.; Chem. Educ. Res. Pract. 2007, 8, 130; Selco, J. I.; Roberts, J. L.; Wacks, D. R.; J. Chem.Educ. 2003, 80, 54; Yingjie, L.; Zaiqun, L.; The China Papers 2003, july, 34.

11. Jones-Wilson, T. M.; J. Coll. Sci. Teach. 2005, 35, 42; Ram, P.; J. Chem. Educ. 1999, 76, 1122.

12. Cruickshank, B.; Olander, J.; J. Coll. Sci. Teach. 2002, 31, 374.

13. Duch, B. J. In The Power of Problem Based Learning. A práctical "How to" for Teaching Undergraduate Courses in Any Discipline; Duch, B. J.; Groh, S. E.; Allen, D. E., eds.; Stylus: Sterling, 2001, p. 39.

14. Hutchinson, J. S.; Univ. Chem. Educ. 2000, 4, 3; http://www.fing.edu. uy/ nmoller/2006/cursotodo.pdf, accedida en Enero 2007; Wood, C.; Chem. Educ. Res. Pract. 2006. 7, 96; http://ublib.buffalo.edu/libraries/ projects/cases/ubcase.htm\#chemistry, accedida en Marzo 2010.

15. http://www.udel.edu/pbl/curric/chem103-prob.html, accedida en Agosto 2007; http://personal.telefonica.terra.es/web/jramonl77/pbl/escritjulio/ abp\%20quimica.pdf, accedida en Agosto 2008; Ward, C.; Chem. Educ. Res. Pract. 2006, 7, 96.

16. Karuktsis, K.; J. Coll. Sci. Teach. 2003, 33, 36.

17. Lehman, J. W., Operational Organic Chemistry: to problem-solving approach to the laboratory course., $3^{\text {rd }}$ ed., Prentice-Hall: New Jersey, 1999.

18. Dai, Y.; The China Papers 2004, November, 6.

19. Belt, S. T.; Evans, E. H.; Mccreedy, T. C.; Overton, T. L.; Summerfield, S.; Univ. Chem. Educ. 2002, 6, 65; Cancilla, D. A.; J. Chem. Educ. 2001, 78, 1652; Wenzet, T.; Austin, R. N.; Environ. Sci. Technol. 2001, 35, 326.

20. Abate, M. A.; Meyer-Stout, P. J.; Stametakis, M. K.; Gannett, P. M.; Dunsworth, T. S.; Nardt, A. H.; Am. J. Pharm. Educ. 2000, 64, 74.

21. Chrobak, R.; Proceedings of 2nd International Conference on Information Technology Based Higher Education and Training [online], Kumamoto, Japan, july 2001, http://www.eecs.kumamoto-u.ac.jp/ITHET01/proc/082. pdf, accedida en Marzo 2010.

22. Llorens, J. A.; Educ. Quím. 2007, 18, 259.

23. Busquets, J.; Roldán, D.; Martínez, S.; Del Blanco, D.; PoliformaT: una estrategia para la formación on-line en la Educación Superior Virtual Educa, Bilbao, 2006; http://ihm.ccadet.unam.mx/virtualeduca2006/ pdf/177-DRM.pdf, accedida en Marzo 2010.

24. Llorens, J. A. En Aprendizaje activo de la física y la química; Pinto, G.; ed.; Equipo Sirius: Madrid, 2007, p. 281-288.

25. Duch, B. J.; Groh, S. E. En The Power of Problem Based Learning. A práctical "How to" for Teaching Undergraduate Courses in Any Discipline; Duch, B. J.; Groh, S. E.; Allen, D. E., eds.; Stylus, Sterling: Virginia, 2001, p. 95. 


\title{
EL APRENDIZAJE BASADO EN PROBLEMAS COMO ESTRATEGIA PARA EL CAMBIO METODOLÓGICO EN LOS TRABAJOS DE LABORATORIO
}

\author{
Juan-Antonio Llorens-Molina \\ Departamento de Química, Escuela Técnica Superior del Medio Rural y Enología, Universidad Politécnica de Valencia, Camino \\ de Vera s/n, 46021 Valencia, España
}

\section{Programa de la asignatura}

\section{Programa de teoría:}

Parte introductoria: Unidad 1: "Introducción a la química orgánica, interpretación de las propiedades físicas desde la estructura molecular”; unidad 2: "Las reacciones orgánicas: aspectos generales y clasificación"; unidad 3: "Hidrocarburos, el petróleo, procesos petroquímicos, normas de formulación y nomenclatura"; unidad 4: "Isomería".

Estudio específico de las principales funciones orgánicas: Unidad 5: "Alquenos y alquinos, polienos, polímeros sintéticos de adición"; unidad 6: "Hidrocarburos aromáticos"; unidad 7: "Derivados halogenados, reacciones de sustitución nucleófila y eliminación y sus mecanismos"; unidad 8: "Alcoholes, fenoles, éteres y epóxidos"; unidad 9: "Aldehídos y cetonas"; unidad 10: "Ácidos carboxílicos y sus derivados"; unidad 11: "Compuestos nitrogenados".

\section{Programa de prácticas:}

(1) Introducción. Manejo de modelos moleculares; (2) Determinación del contenido en azúcares de la algarroba mediante un método refractométríco. Determinación del contenido en cafeína de té por extracción líquido-líquido, (3) Obtención de aceites esenciales de plantas aromáticas mediante destilación por arrastre de vapor de agua (Equipo Clavenger); (4) Determinación del contenido en grasa del cacahuete mediante extracción sólido-líquido con equipo Soxhlet; (5) Obtención de jabón y biodiesel. Reacciones de saponificación y transesterificación; (6) Síntesis y purificación del ácido acetilsalicílico; (7) Separación e identificación de los componentes de la acidez fija del vino por cromatografía sobre papel.

\section{Problemas experimentados a lo largo de los tres cursos}

Los criterios que dieron lugar a la eliminación de ciertos problemas tras el primer curso fueron:

(1) La escasa aportación del problema al contenido de la asignatura, bien por su excesiva complejidad (problemas 10 y 11) o por la pobreza teórica de su desarrollo, al dar lugar a simples comprobaciones experimentales (problemas 9 y 13); (2) Dificultad en el tratamiento experimental, ya que ciertas técnicas no admiten simplificaciones que sean compatibles con resultados claros que permitan abordar el problema con el rigor necesario $(10,11,12)$; (3) En otros casos (problemas 8 y 14), la dificultad radica en la propia formulación del problema, ya que da lugar a un planteamiento excesivamente puntual que no exige al grupo un desarrollo posterior de la suficiente entidad. Por otra parte, en algunas de las actividades el primer año el grado de conexión con la vida real y el carácter multidisciplinar no mostró ser lo suficientemente evidente y, de hecho, fueron eliminadas $(8,13$ y 14) o reformuladas (la 16 procede de la 6)

Descripción de un ejemplo acerca de la obtención de biodiesel a partir de residuos de grasas domésticas

\section{Actividad inicial (aspectos explícitos)}

El escenario del problema es la hipotética creación por parte

*e-mail: juallom2@qim.upv.es del grupo de estudiantes de una empresa destinada a la obtención de biodiesel a partir de residuos de grasas domésticas. La posterior delimitación del problema va dirigida hacia la estimación aproximada de la entalpía de combustión del biodiesel obtenido como característica especialmente relevante en cuanto a su uso como combustible en locomoción.

Los principales retos planteados al alumnado son: (1) identificar las principales variables que permiten evaluar la utilidad de un combustible para automoción; (2) proponer y utilizar un método basado en una aplicación informática sencilla para el cálculo de dichas variables; (3) proponer y desarrollar un proceso experimental a escala de laboratorio para obtener biodiesel a partir de residuos domésticos de grasas y evaluar su rendimiento; (4) valorar las dificultades y posibles alternativas para la puesta en marcha de un proyecto basado en la fabricación y comercialización de este combustible. Puede accederse a la actividad inicial propuesta a través del siguiente enlace: https:// poliformat.upv.es/access/content/user/19877419/Biodiesel 09.doc

\section{Guía para la puesta en marcha y desarrollo de la actividad} (aspectos implícitos).

Consiste en el conjunto de reflexiones que debe servir de guía al profesorado para conducir la entrevista inicial; en definitiva, se trata de prever los recursos que debemos tener preparados para que, fruto de esta primera discusión, surja un primer plan de trabajo para el desarrollo y resolución del problema. Estas reflexiones, tal como se puede apreciar en la Figura 1S, constituyen el inicio del proceso de autorregulación-feed-back que tiene lugar a lo largo de la actividad.

\section{Breve descripción de otros ejemplos de actividades experimentadas}

Para expresar el significado y dinámica de esta propuesta, puede ser útil describir brevemente otras actividades representativas. Por ejemplo, la investigación sobre los factores que determinan la amplia variabilidad en los precios de aceites esenciales (Problema 3) es de gran relevancia, ya que la consideración del origen geográfico, factores edafoclimáticos, variedad botánica, la diversidad de quimiotipos, el tipo de cultivo, ecológico o convencional, etc., aportan un enriquecedor enfoque multidisciplinar. Por otra parte, tal como está planteado el problema, la influencia de la composición química en el precio no se refiere solamente a la mayor o menor presencia de ciertos compuestos que en los aceites esenciales de origen natural afectan decisivamente a su precio (el linalool en el aceite de lavanda, por ejemplo), sino que también se refiere a la comercialización de disoluciones de dichos aceites en disolventes (polialcoholes, generalmente), muy populares al hallarse a la venta habitual en muchos comercios y bazares. Ello permite a los estudiantes abordar problemas de interés profesional como la calidad de los productos y su normativa, el problema de las adulteraciones, etc.

Otra actividad cuyo desarrollo fue muy fructífero es la referida a la aplicación de productos naturales en el almacenamiento y conservación de las patatas (Problema 15). La búsqueda de alternativas naturales al tratamiento químico tradicional en el tratamiento post-cosecha de tubér- 


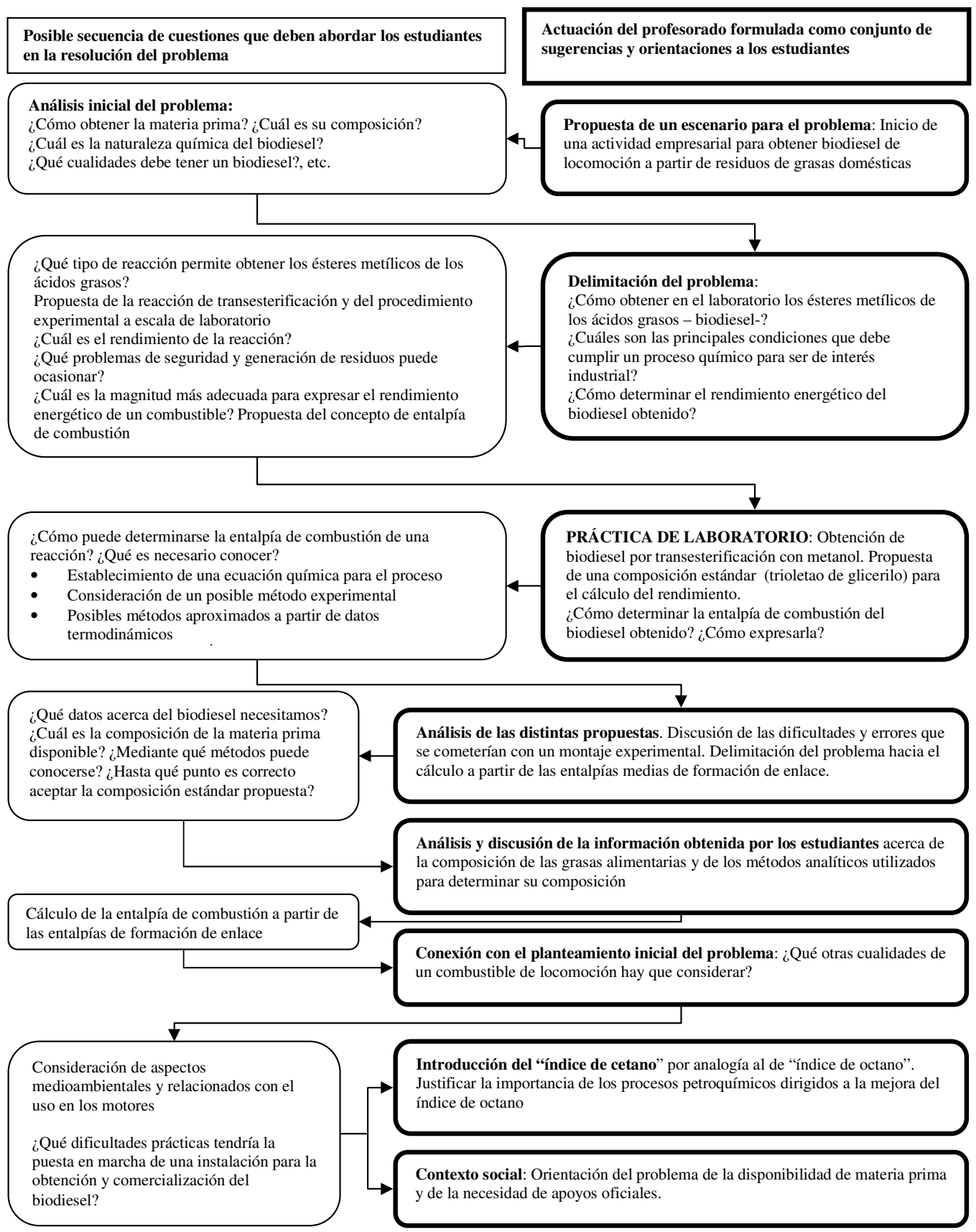

Figura 1S. Proceso de autorregulación y feed-back en el problema relacionado con la obtención de biodiesel

culos es fácilmente accesible en la red y conduce a la consideración de la actividad biológica de los aceites esenciales. La elección de la patata no es, ni mucho menos, irrelevante, ya que la información disponible en la red dirige a los estudiantes a un principio activo: la carvona, cetona terpénica, ópticamente activa, que bajo sus dos formas enantioméricas $\mathrm{R}$ y $\mathrm{S}$, es particularmente abundante en los aceites esenciales de Mentha spicata, L. y Carum carvi, L., respectivamente. La importancia biológica de las posibles diferencias de actividad biológica entre enantiómeros es importante desde el punto de vista de las relaciones ciencia-técnicasociedad (recordemos el problema de los efectos teratogénicos de la $(S)$ talidomida). En el marco de esta actividad, el diseño de un experimento para evaluar comparativamente la eficacia de los dos enantiómeros de la carvona plantea un conjunto de problemas que configuran todo un proceso de investigación dirigida: (1) cómo obtener el aceite esencial y verificar su composición; (2) cómo aplicar el aceite y con qué concen- tración; (3) cómo cuantificar la germinación de los tubérculos; (4) cómo realizar el seguimiento y la aplicación analizando diferentes alternativas: una sola dosis inicial y estudiar el inicio de la germinación, tratamiento prolongado y estudiar su evolución..., etc.

El problema 5: “¿Contiene cafeína el “té descafeínado?” es en realidad la prolongación de una de las prácticas de laboratorio, pero exige al grupo que lo desarrolla una aproximación y evaluación comparativa, sobre todo, desde el punto de vista medioambiental, de los métodos de extracción industrial de la cafeína (extracción con diclorometano y $\mathrm{CO}_{2}$ supercrítico) que al final deberán explicar brevemente al resto de sus compañeros; al mismo tiempo, les exige también buscar métodos de identificación basados en pruebas químicas sencillas y rápidas (en este caso, el test de la murexida, específico de la estructura de la purina). Ello incide en uno de los aspectos conceptuales básicos del curso: la relación entre reactividad y estructura. 
La posibilidad de rentabilizar los residuos de la producción de la cera de abejas (problema 17) también mostró una gran potencialidad. Este problema fue originariamente una consulta profesional formulada al profesor por una empresa apícola, pero dados los conceptos y métodos implicados, pareció accesible a su tratamiento por los estudiantes. Y así fue en realidad, ya que consiguieron poner en juego, de manera autónoma, un protocolo experimental basado en la aplicación de técnicas aprendidas previamente en una de las prácticas (manejo del equipo Soxhlet), por otra parte, tuvieron que fundamentar dicho protocolo en la consideración del disolvente adecuado a la naturaleza apolar de la sustancia que debían extraer. También se observa en este caso cómo subyace en el problema otro de los aspectos conceptuales clave del curso: la relación estructura molecular-fuerzas intermoleculares-propiedades físicas. Al mismo tiempo, se halla presente, una vez más, la perspectiva multidisciplinar, ya que el enfoque inicial del problema les obliga a considerar la variedad de productos que puede haber en los residuos de la cera.

En otras ocasiones, lo más relevante no es tanto la resolución del problema en sí como algunos de los procesos implicados. Es lo que ocurre, por ejemplo, en el 8 (“¿Grasas saturadas o insaturadas?”). La aplicación de la reacción de adición electrófila con $\mathrm{Br}_{2}$ para determinar la presencia de instauraciones es fácilmente identificada, ya que es una de las reacciones importantes del temario; sin embargo, como no se les proporciona dicho reactivo por motivos de seguridad, deben hallar, por si mismos, recurriendo a los conocimientos previos del cuatrimestre anterior, un proceso para obtenerlo (el proceso al que finalmente se llega es consiste en acidificar una disolución de hipoclorito de sodio para obtener $\mathrm{Cl}_{2}(\mathrm{aq})$, hacer reaccionar éste con $\mathrm{Br}$, oxidándolo a $\mathrm{Br}_{2}$ que es extraído posteriormente con $\mathrm{CCl}_{4}$ )

Encuesta aplicada a los estudiantes para la evaluación de la actividad, con sus resultados (Tabela 2)

Tabla 1S. Enumeración de los problemas experimentados a lo largo de los tres cursos

Relación de problemas planteados el primer curso de experimentación

Se mantuvieron:

1. Un producto aromatizante desconocido ¿Cómo podemos identificarlo y obtenerlo?

- Análisis elemental de sustancias

- Ésteres. Propiedades de los ésteres

- Reacciones de esterificación

2. Estimación del calor de combustión de un biodiesel

- Entalpías de reacción, combustión y formación de enlace

- Estructura de las grasas

- Reacciones de transesterificación.

3. ¿Por qué tanta diferencia de precio? Relación precio-composición en aceites esenciales empleados en aromaterapia

- Grupos funcionales

- Terpenos y terpenoides

- Cromatografía de gases

4. ¿Cómo podemos distinguir aspirina $y$ paracetamol?

- Fenoles. Reconocimiento de fenoles.

- Reacciones de transferencia de grupo acilo.

- Identificación, aislamiento y aplicación de principios activos en la industria farmacéutica.

5. ¿Contiene cafeína el "té descafeinado"?

- Procesos de extracción sólido-líquido y líquidolíquido.

- Estructura y reconocimiento de los alcaloides.

6. Identificar para separar. Separar para reciclar

- Estructura y propiedades de los polímeros más importantes.

- Polímeros de adición y condensación.

- Reconocimiento de polímeros.

7. ¿Grasas saturadas o insaturadas?

- Procesos redox. Características redox de los halógenos. Extracción líquido-líquido.

- Reacciones de adición electrofílica.
Se eliminaron:

8. ¿Por qué hay plásticos rígidos y termoestables mientras que otros son flexibles y fáciles de moldear al calentarlos?

- Relación estructura-propiedades

- Propiedades mecánicas y térmicas de los polímeros.

- Reacciones de polimerización.

9. ¿Qué ácido contiene el "agret" (Oxalys pescaprae)?

- Ácidos carboxílicos

- Cromatografía plana (papel y TLC)

10. El color de las flores

- Pigmentos vegetales. Antocianinas. Estructura.

- Concepto de par conjugado ácido-base. pH. Indicadores ácido-base.

11. ¿Cómo actúa la saliva en los alimentos?

- Catálisis. Enzimas. Hidratos de carbono.

- Reacciones de hidrólisis

- Reconocimiento de azúcares reductores. Ensayos de Felhing y Benedict.

12. ¿Cómo puede medirse el grado de alcoholemia en un conductor?

- Procesos redox y valoraciones. Influencia de la acidez.

- Reacciones de oxidación de alcoholes.

13. ¿De qué tipo de sustancia se trata?

- Reconocimiento de grupos funcionales mediante reacciones características.

14. Nilón y Kevlar. ¿Cómo la estructura de la materia explica sus propiedades prácticas?

- Fuerzas intermoleculares.

- Relación entre estructura de un polímero y propiedades mecánicas.

- Manejo de modelos moleculares
Problemas introducidos durante el segundo y tercer curso.

15. ¿Cómo podemos prevenir el "grillado" de las patatas?

- Tensoactividad y agentes emulsionantes

- Terpenos y derivados. Actividad biológica

16. Fabricación de macetas de plástico reciclado: ¿Podemos utilizar los residuos domésticos de plásticos?

- Tipos de polímeros y técnicas sencillas para su reconocimiento. Reciclaje.

17. ¿Podemos optimizar el rendimiento en la obtención de cera de abejas tratando los residuos procedentes de su producción?

- Extracción sólido-líquido

- Equipo Soxhlet.

- Estructura y solubilidad de las sustancias.

18. ¿Cuándo está madura la fruta?

- Índice de madurez.

- Ácidos presentes en las frutas. Valoraciones ácido-base.

- Refractometría. ${ }^{\circ}$ Brix. 
Tabla 2S. Encuesta aplicada para la evaluación de las actividades por los estudiantes

Categorías

$05 / 06 \%$

$07 / 08 \%$

1. Al asignar el trabajo, se proporcionó un documento escrito y se mantuvo una entrevista con el grupo. Estas orientaciones iniciales las consideras...

a) Insuficientes. Fuimos incapaces de ponernos a trabajar.

b) Adecuadas. Obtuvimos la información justa para iniciar el análisis del problema.

c) Excesiva. Parte de la información la habríamos obtenido nosotros mismos

2. Durante la resolución del problema se ha procurado atender a los grupos de modo que su trabajo tuviera un elevado grado de creatividad y autonomía. ¿Cómo crees que se ha desarrollado realmente el trabajo?

a) Ha sido como una receta. No se ha favorecido la autonomía e iniciativa del grupo.

b) Ha habido cierta autonomía e iniciativa, pero se ha proporcionado excesiva información y se han resuelto dudas y situaciones que hubiera superado el grupo.

c) La información y orientaciones proporcionadas han sido las adecuadas para desarrollar nuestro trabajo con un elevado grado de autonomía.

d) La información y orientaciones proporcionadas han sido insuficientes y nos hemos encontrado frecuentemente perdidos durante el desarrollo del problema.

3. El problema se planteó para ser desarrollado en equipo. ¿Cuál de las siguientes afirmaciones refleja mejor el método de trabajo seguido en el grupo?

a) El trabajo ha sido repartido y realizado individualmente, con muy poca colaboración

b) El trabajo ha sido repartido y realizado individualmente pero al menos, al principio y al final, hay un encuentro para unificar criterios, intercambiar información, etc.

c) El grupo siempre ha trabajado colectivamente.

4. Sobre el ritmo de trabajo:

a) El problema fue analizado y desarrollado precipitadamente las dos últimas semanas de curso.

b) El trabajo comenzó a realizarse hace más de un mes, pero de una manera irregular.

c) El trabajo ha sido desarrollado de una manera constante y metódica (una sesión semanal como mínimo)

5. El tema desarrollado te ha parecido...

a) Poco interesante. No ha aportado nada significativo a mi formación en la asignatura

b) Interesante, pero ha aportado poco a la asignatura

c) En cuanto al programa del curso ha sido un apoyo, pero no me ha interesado demasiado

d) El tema me ha parecido interesante y ha contribuido a mejorar la comprensión y aprendizaje del algún aspecto de la asignatura.

6. El grado de conocimientos básicos para abordar el problema lo considero..

a) Insuficiente. No he entendido términos y conceptos básicos.

b) Suficiente. El tema desarrollado está adaptado al nivel del curso.

c) El nivel ha sido bajo respecto a los contenidos del curso.

7. ¿Cómo valoras la contribución de este trabajo a la nota del curso:

a) Insuficiente

b) Adecuada

c) Excesiva

8. La información utilizada ha sido obtenida de..

a) Internet, principalmente

b) Manuales y libros de texto, principalmente.

c) Diversas fuentes: internet, libros, revistas, apuntes, etc.

d) Otras fuentes

9. El grado de motivación que has experimentado con esta forma de trabajar ha sido...

a) Menor que hacia el resto de actividades de la asignatura: clases, autoevaluaciones, etc.

b) Similar
0

92

3

\section{0}

0

